\title{
Die Eigenschaften der Online-Kommunikation
}

2.1 Erscheinungsformen der Online-Kommunikation - 12

2.2 Der Kommunikationsinhalt und die Schwierigkeit der Interpretation - 14

2.3 Fehlendes Hintergrundwissen und (in-)adäquates Verhalten - 17

2.4 Die Rolle von Bildern in der Online-Kommunikation - 19

2.5 Das digitale Datenformat und seine psychologischen Folgen - 21

2.6 In der Praxis: Wie sich die Eigenschaften der Online-Medien auswirken - 28

2.7 Zusammenfassung: Die Eigenschaften der Online-Kommunikation und ihre Vor- und Nachteile - 35 
Döring, N. (2003). Sozialpsychologie des Internet. Göttingen: Hogrefe.
$>$ Lesen Sie in diesem Kapitel:

- Welche spezifischen Vor- und Nachteile die Eigenschaften der Online-Medien mit sich bringen,

- welche unterschiedlichen Aufgaben Text und Bilder in der Online-Kommunikation haben,

- warum im Online-Dialog ein erhöhtes Potenzial für Missverständnisse besteht und wie man damit umgehen kann,

- wie sich Online-Kommunikation auf bestehende Hierarchien auswirkt und was dies für Führungskräfte bedeutet und

- was man im Online-Kontakt mit anderen Kulturen beachten sollte.

In Kommunikations- oder Medientrainings werden oft psychologische Prozesse erläutert, weil sie die Kommunikation in unterschiedlicher Weise beeinflussen. Diese Verwandtschaft von Kommunikation und Psychologie gilt für den Online-Bereich genauso. Auch wenn es heute noch etwas ungewohnt ist, von einem Online-Medientraining zu reden, gibt es doch Aspekte und Themen des Internets, die man kennen sollte, wenn man die Kommunikation erfolgreich gestalten will. In den nun folgenden Kapiteln 2-4 lernen Sie Online-Medien aus drei verschiedenen psychologischen Perspektiven kennen:

- In diesem Kap. 2 die spezifischen Eigenarten der Online-Medien und ihre psychologische Relevanz.

- In $\triangleright$ Kap. 3 die Perspektive auf den Nutzer, der für bestimmte Kommunikationsaufgaben bestimmte Medien auswählt.

- In $\triangleright$ Kap. 4 folgt der Blick auf die sozialen Gruppen, die sich im Internet bilden, und ihre Merkmale und Gesetzmäßigkeiten.

Diese Aufteilung erfolgt in Anlehnung an das Standardwerk von Döring (2003), das einen umfassenden Überblick über die psychologische OnlineForschung verschafft.

\subsection{Erscheinungsformen der Online- Kommunikation}

Bevor von den Eigenschaften gesprochen wird, lohnt es sich, erst einmal die verschiedenen Erscheinungsformen systematisch anzuschauen. Im Laufe der Jahre hat sich eine beachtliche Vielfalt entwickelt. Anhand dieser Auflistung können auch bereits gewisse Merkmale definiert und abgegrenzt werden (- Tab. 2.1).

- Tabelle 2.1 zeigt, dass sich die Online-Dienste grob in zwei Gruppen aufteilen lassen: die synchronen und die asynchronen Dienste. Synchron heißt, dass mindestens zwei Personen zeitgleich online sind. Asynchron ist die zeitlich versetzte Online-Kommunikation. Gerade die Asynchronizität 
- Tab. 2.1. Erscheinungsformen der Online-Kommunikation. (Nach Döring, 2003, S. 125, mit eigenen Ergänzungen)

\begin{tabular}{|c|c|c|}
\hline $\begin{array}{l}\text { Kommunikations- } \\
\text { typ }\end{array}$ & $\begin{array}{l}\text { Asynchroner } \\
\text { Internet-Dienst }\end{array}$ & $\begin{array}{l}\text { Synchroner } \\
\text { Internet-Dienst }\end{array}$ \\
\hline $\begin{array}{l}\text { Individuelle } \\
\text { Kommunikation } \\
(1: 1)\end{array}$ & E-Mail & $\begin{array}{l}\text { Internet-Telefonie } \\
\text { Instant Messaging }\end{array}$ \\
\hline $\begin{array}{l}\text { Gruppen- } \\
\text { kommunikation } \\
(n: n)\end{array}$ & $\begin{array}{l}\text { Mailinglisten } \\
\text { Newsgroups } \\
\text { Messageboards } \\
\text { Diskussionsforen } \\
\text { Weblogs (Blogs) }\end{array}$ & $\begin{array}{l}\text { Chats: IRC, webbasiert, } \\
\text { grafisch } \\
\text { Internet-Videokonferenzen } \\
\text { Online-Spiele } \\
\text { Online-Rollenspiele: text- } \\
\text { basiert (MUDs) oder gra- } \\
\text { phisch (MMORPGs) } \\
\text { Online-Tauschbörsen }\end{array}$ \\
\hline $\begin{array}{l}\text { Uni- oder Massen- } \\
\text { kommunikation } \\
(1: n)\end{array}$ & $\begin{array}{l}\text { Websites } \\
\text { Online-Videos } \\
\text { Weblogs (Blogs), } \\
\quad \text { Video-Blogs (Vlogs) } \\
\text { Podcasts }\end{array}$ & $\begin{array}{l}\text { Livevideos (auf Websites), } \\
\text { Firmenfernsehen (Busi- } \\
\text { ness TV) }\end{array}$ \\
\hline
\end{tabular}

hat z. B. der E-Mail-Kommunikation zu ihrem rasanten Aufstieg verholfen: Kein anderes Medium hat bisher die Möglichkeit geboten, nachts um 23.30 Uhr eine Nachricht abzusenden, ohne den Empfänger zu belästigen, der die Nachricht am nächsten Morgen um 6 Uhr, oder wann auch immer er will, in der Mailbox abrufen kann. Aber auch Newsgroups und Blogs leben von der schnellen und einfachen Möglichkeit des asynchronen Informationsaustausches.

Synchrone Kommunikation findet nur statt, wenn mehrere Personen gleichzeitig online sind. Sie bietet eine faszinierende Art von Lebendigkeit: Weil Online-Partner schnell reagieren, herrscht eine gesprächsnahe Atmosphäre, die das eigene Verhalten und Empfinden trotz fehlender Sinnesreize maßgeblich beeinflussen kann. Der Übergang zwischen asynchron und synchron ist manchmal fließend. Auch E-Mails und Newsforen können - bei rascher Reaktionszeit des Gegenübers - fast synchronen Charakter haben.

Auf der anderen Achse werden drei verschiedene Arten von Kommunikationspartnern $(1: 1,1: \mathrm{n}, \mathrm{n}: \mathrm{n})$ definiert. Bemerkenswert ist, dass die Massenkommunikation $(1: \mathrm{n})$ nicht nur von einer »Institution « (Fernsehanstalt, Verlag) ausgehen kann, wie das traditionellerweise der Fall war, sondern auch von einer einzelnen, unbekannten Person, die nur über marginale Infrastruktur verfügt. Hier hat das Internet die Kommunikation revolutioniert, denn durch diese $1: \mathrm{n}$-Kommunikation entsteht eine ganz neue Dynamik, die gerade auch für Unternehmen von Bedeutung ist.
Massenkommunikation ist nicht mehr nur Institutionen vorbehalten 
Die Ausdrucksfähigkeit ist online eingeschränkt

\subsection{Der Kommunikationsinhalt und die Schwierigkeit der Interpretation}

\section{Beispiel}

Falsche Interpretation

»lch habe vor einiger Zeit einen Fall gehabt, als ein Außendienstmitarbeitender eine komplizierte Offerte hätte schreiben sollen. Ich habe ihm dann per E-Mail mitgeteilt, er solle mir die Unterlagen schicken, ich würde die Offerte schreiben. Zwei Tage später ist er gekommen und verlangte ein Gespräch, weil er nicht erkannt hatte, dass ich ihm helfen wollte. $\mathrm{Er}$ hatte das Gefühl, ich wolle inn auf die Seite schieben und habe vor, ihn zu entlassen."

Sales Manager, international tätiges Unternehmen für Arbeitsschutzprodukte, $m, 58$.

Ein großer Teil der Online-Kommunikation findet textbasiert statt - zumindest bis zum heutigen Zeitpunkt. Diese Reduktion auf Buchstaben führt zu einer Reduktion der Ausdrucksfähigkeit - da man eben »nur" Text zur Verfügung hat, um eine Botschaft weiterzugeben. Gerade diese Einschränkung wird häufig als negativer Aspekt der neuen Medien hervorgehoben, der zu einem verantwortungslosen Umgang mit anderen und zu mehr Oberflächlichkeit im zwischenmenschlichen Kontakt führe. Dies ist eine falsche und undifferenzierte Pauschalisierung. Auch die schriftliche Online-Kommunikation kennt Zwischentöne, und eine textbasierte Kommunikationsform ist - je nach Aufgabenstellung - besser geeignet für die Übermittlung einer Nachricht ( Kap. 3). Dennoch entstehen durch die Textbasiertheit spezifische Problemstellungen, die im Folgenden erläutert werden.

\subsubsection{Ent-Emotionalisierung}

Wenn ich einer Person »face to face « gegenüberstehe und ihr etwas Schwieriges mitteile, übermittle ich nicht nur Informationen über den verbalen Kanal, sondern auch über den paraverbalen (Stimmlage, Wortwahl) und den nonverbalen (Körpersprache) Kanal. Die Person sieht, wie ich die Stirn runzle, hört, wie ich die Stimme senke und seufze, und sie merkt, wie sehr ich jedes Wort abwäge, bevor ich es verwende. Bei einer E-Mail findet der Austausch ausschließlich auf der Ebene der geschriebenen Sprache statt. Man spricht deshalb von einer Kanalreduktion. Mit dem Aufkommen der computervermittelten Kommunikation wurde dieser Sachverhalt in den 80er- und 90er-Jahren ausführlich diskutiert und kritisiert. Man sprach von Ent-Sinnlichung, Ent-Emotionalsierung und sogar von EntMenschlichung, und man befürchtete, dadurch würde die psychosoziale Ebene des Austausches gänzlich verkümmern (s. auch Winterhoff-Spurk \& Vitouch, 1989; Mettler-von Meibom, 1994). 
Mit der Online-Erfahrung von mehr als einem Jahrzehnt lässt sich sagen, dass diese Befürchtungen sich nicht bestätigt haben. Die meisten von uns verwenden heute E-Mail und Internet, ohne dabei psychisch Schaden zu nehmen.

Und dennoch ist an der Kritik etwas dran: denn sie legt den Finger auf jene Schwierigkeiten, denen wir in der Online-Kommunikation begegnen und die uns vorher in dieser Ausprägung unbekannt waren. Wenn wir z. B. - wie im eingangs erwähnten Beispiel - plötzlich einen total verstörten Mitarbeiter am anderen Ende der Netzwerkverbindung haben und gar nicht verstehen, warum dieser so aus der Fassung geraten ist.

\subsubsection{Interpretationsspielraum}

Warum eine freundlich gemeinte und knapp verfasste E-Mail so viel auslösen kann, erklären zwei Kommunikationsregeln von Watzlawick et al. $(1969,2000)$.

\section{Man kann nicht nicht kommunizieren (1. Axiom)}

Diese Regel ist darauf begründet, dass es zu »Verhalten« kein Gegenteil gibt. Das heißt, wie auch immer man sich benimmt, macht man damit eine Aussage. Wenn man schweigt und sich abwendet, heißt das z. B., dass man in Ruhe gelassen werden will. Oder allgemeiner ausgedrückt: Auch wenn ich schweige, sage ich etwas aus. Nur ist diese Art von Aussage viel schwieriger zu deuten, als wenn ich eine explizite Botschaft sende.

\section{Jede Nachricht hat eine Inhalts- und eine Beziehungsebene (2. Axiom)}

Wann immer wir kommunizieren, sagen wir damit etwas über unsere Beziehung zum Kommunikationspartner aus. Die Beziehungsebene ist sogar prägend dafür, wie wir den Inhalt der Kommunikation interpretieren. Beispiel: Die Frage »Sind das echte Perlen?« kann Neid, Staunen oder Respektlosigkeit ausdrücken. Auch wenn der Fragende vielleicht Staunen zum Ausdruck bringen wollte, ist nicht gesagt, dass die Gefragte das auch so interpretiert.

In der Face-to-face-Kommunikation haben beide Partner Gelegenheit, zur richtigen Interpretation der Beziehungsebene beizutragen und Missverständnisse schnell aus dem Weg zu räumen (- Abb. 2.1). In der OnlineWelt ist das aber sehr schwierig, da die unmittelbare Reaktion des Empfängers nicht sichtbar ist. Es ist ja nicht so, dass wir absichtlich »schweigen«. Aber die (para- und nonverbalen) Kanäle, die wir online nicht aktiv mit Informationen bedienen, lassen der Person auf der Empfängerseite großen Interpretationsspielraum. Das kann besonders dann zu Problemen führen, wenn unterschwellig bereits ein Konfliktpotenzial vorhanden ist. Die Interpretation wird dann von früheren Erfahrungen, schlechten Erwartungen und Ängsten geprägt, und auch wenn wir gute Absichten haben, werden uns sehr rasch schlechte unterstellt.
Schriftbasierte

Kommunikation bietet Interpretationsspielraum

Watzlawicks

Kommunikationsregeln 
- Abb. 2.1. Fehlende paraund nonverbale Informationen führen zu Missverständnissen

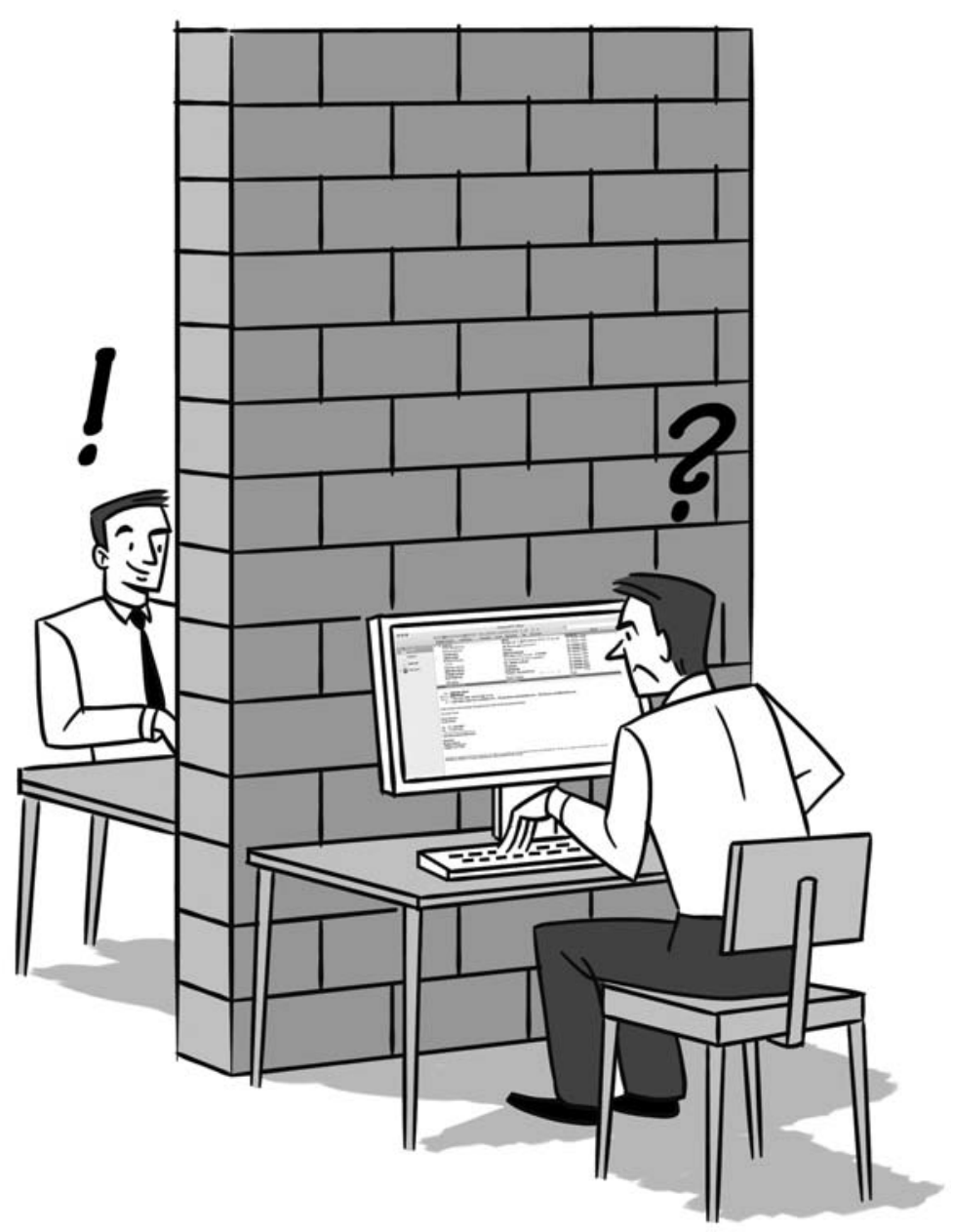

In jeder Online-Nachricht sind ein Inhalts- und ein Beziehungsaspekt vorhanden. Das Fehlen von para- und nonverbalen Gesprächsinformationen erschwert die korrekte Interpretation des Beziehungsaspekts. Darum wird die Botschaft vom Empfänger gemäß seiner Erfahrungen, seines Vorwissens und seiner aktuellen Stimmung interpretiert - zu Gunsten oder Ungunsten des Senders.

Darum sollten wir uns immer genau überlegen, wen wir als Empfänger einer Online-Botschaft vor uns haben.

(? Wenn das Verhältnis zwischen Kommunikationspartnern durch Misstrauen oder Konkurrenzdenken geprägt ist oder zwischen ihnen ein starkes Machtgefälle besteht, erhöht sich die Bereitschaft des Empfängers zur negativen Interpretation einer Botschaft.

Die Internet-Gemeinschaft ist sich dieses Konfliktpotenzials durchaus bewusst. Im Netz sind deshalb unzählige Netiquettes zu finden, die Verhaltensregeln bieten und vor den drohenden Gefahren warnen. Dies sind 
wirklich nützliche Instrumente, die man auch für den privaten E-MailVerkehr verwenden kann. Mehr zur professionellen E-Mail-Kommunikation $>$ Kap. 7.

\subsubsection{Effiziente und "schlanke" Informationsübermittlung}

Die Kanalreduktion hat aber auch handfeste Vorteile. Durch den Wegfall der "Zwischentöne" wird die Informationsübermittlung effizient und schnell. Alle textbasierten Medien profitieren von diesem Effekt:

- Zeitungslesen ist für die Vermittlung detaillierter Sachverhalte effizienter als Fernsehen, weil Inhalte schriftlich präziser beschrieben werden können.

- Schriftliche Online-Newsdienste sind effizienter als Podcasts, weil man die wichtigsten News lesend schneller im Überblick hat als hörend.

etc.

Natürlich kommt es stark darauf an, welche Art von Botschaft man zu transportieren hat. Nicht immer ist die sachbezogene, effiziente Informationsvermittlung das Hauptkriterium. Als Beispiel sei hier Business TV angeführt, das man z. B. dazu einsetzen kann, in der internen Kommunikation den Mitarbeitenden die Jahresziele zu erläutern. Durch die persönliche Präsenz des Topmanagements (wenn auch nur am Bildschirm) kann man Nähe zu den Mitarbeitenden zeigen und Vertrauensbildungsprozesse unterstützen. Dies geschieht jedoch immer auf Kosten der Effizienz, denn die Angestellten müssen, wenn sie alle Unternehmensziele kennen wollen, die Sendung von A-Z schauen. (Außerdem könnte es sein, dass sie durch eine unpassende Krawatte des Finanzchefs oder einen nervösen Tick des CEOs vom Inhalt abgelenkt werden.) Es gilt also, immer abzuwägen, welche Anteile man höher gewichten bzw. welches Ziel man in erster Linie erreichen will.

Für schnelle, detaillierte und rein sachliche Informationsübermittlung eignet sich die kanalreduzierte, textbasierte Kommunikationsform besser als die »kanalreiche«, die mit Ton und Bildern arbeitet.

Mehr zu diesem Thema $\triangleright$ Kap. 3, das sich mit der Medienwahl befasst.

\subsection{Fehlendes Hintergrundwissen und (in-)adäquates Verhalten}

\subsubsection{Enthemmung}

Die Vertreter der Filtertheorien sehen Online-Kommunikation nicht als grundsätzlich verarmt und problematisch, sondern legen den Fokus darauf, dass online weniger Information zum sozialen und soziodemografischen Hintergrund des Gegenübers vorhanden ist (Dubrovsky, Kiesler \& Sethna, 1991). Gerade diese Informationen sind aber entscheidend für das
Online-Verhaltensregeln: de.wikipedia.org/wiki/ Netiquette 
Online geben Menschen mehr von sich preis als offline
Unfreundliches OnlineVerhalten ist teilweise systembedingt
Verhalten und die Einstellung gegenüber einer Person. Wenn sich jemand z. B. sehr gut kleidet oder diese Person vom Fernsehen als Top-Managerin bekannt ist, benimmt man sich ihr gegenüber sehr wahrscheinlich respektvoller, als wenn sie in zerrissenen Kleidern und ungepflegt daherkommt.

Dieses Hintergrundwissen fällt online fast gänzlich weg. Einzig das Bildungsniveau kann man anhand des Schreibstils und der Fehlerfreiheit eines Textes einigermaßen abschätzen. Alle anderen Hinweise (sozialer Status, Alter, Geschlecht, Aussehen etc.) fehlen aber erst einmal, wenn das Gegenüber sie nicht aktiv mitteilt.

Das Filtermodell besagt nun, dass durch das Fehlen dieser soziodemografischen Informationen Kontrollmechanismen abgebaut werden und ein enthemmender Effekt entsteht. Man ist offener, ehrlicher als im realen Leben, Hierarchien werden (zumindest vordergründig) nivelliert, die Partizipation aller wird gefördert. Man kennt diese Wirkung von der »Stranger-on-the-train«-Situation, denn einer Person, die man nicht kennt und vermutlich auch nie wieder sieht, vertraut man viel schneller intime Geheimnisse an als dem Nachbarn oder einer Arbeitskollegin. Von dieser Anonymität (und der Faszination dafür) profitieren auch Diskussionsforen, Chats und Blogs im Internet.

Die Bereitschaft zu größerer Offenheit kann in der Online-Beratung (z. B. Kaufberatung, Unterstützung bei spezifischen Themen) große Vorteile haben. In gewissen Bereichen sind Menschen unter der Voraussetzung der Anonymität viel eher bereit, über ihre persönlichen Anliegen zu sprechen (z. B. medizinische oder Gesundheitsthemen). Hier kann man sich die Eigenschaften der Online-Medien zunutze machen und einen neuen Kundenkreis gewinnen, den man anders schwer erreicht hätte.

\section{(. Firmen, die Online-Beratung anbieten, sollten schon beim Aufbau dieses Angebots klar definieren, ob und unter welchen Umständen mit den Rat suchenden Personen auch Kontakte außerhalb der Ano- nymität stattfinden, und diese Bereiche klar trennen (z. B. anonymer Beratungsbereich vs. Online-Shop).}

So können sie verhindern, dass Kunden sich eine Blöße geben und mit Ärger auf die Firma reagieren, falls sie sich aufgrund der Enthemmung (zu) sehr geöffnet haben und das im Nachhinein bereuen.

Auf jeden Fall aber müssen Online-Berater den potentiellen Kunden auf der Website die absolute Vertraulichkeit der preisgegebenen Informationen zusichern und auch technisch die Voraussetzungen für diese Vertraulichkeit schaffen (Verschlüsselung, Anonymisierung). Mehr zu Be-

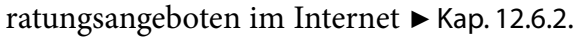

\subsubsection{Aggressives Verhalten}

Die Kehrseite der größeren Offenheit ist auch ein verstärktes aggressives Potenzial der Online-Kommunikation. Durch die Anonymität ist die Sicherheit gegeben, dass man nicht mit Sanktionen rechnen muss, wenn man sich ausfällig verhält. Weil soziale Informationen über eine Person 
fehlen, fällt es schwerer, sich diese Person überhaupt auszumalen und sich vorzustellen, wie sie reagiert, wenn man sie beleidigt.

Offenkundiger Ausdruck dieses aggressiven Verhaltens sind verletzende E-Mails oder Forenbeiträge, in denen jemand bloßgestellt wird. Döring (2003) zählt aber z. B. auch das Verbreiten von Computerviren oder von Spam zum antisozialen Benehmen im Netz.

Die Filtertheorien lassen außer Acht, dass ein großer Teil der OnlineKommunikation nicht mit Fremden stattfindet, sondern mit Menschen, die wir kennen oder in irgendeiner Weise einem sozialen Hintergrund zuordnen können. Arbeitskollegen in Übersee können wir etwas besser als gänzlich Fremde einschätzen, weil sie in der gleichen Firma wie wir arbeiten. Kunden verraten zumindest teilweise etwas über sich durch ihr Interesse an unseren Produkten. Dies nimmt dem enthemmenden Effekt und dem Aggressionspotenzial einen Teil der Brisanz.

Dennoch erleben wir immer wieder Situationen, in denen wir mit dem enthemmenden Effekt konfrontiert sind. Gerade im Kundenkontakt mit Unbekannten, wenn wir z. B. schnoddrig geschriebene Kundenreklamationen per E-Mail erhalten. Es ist daher nützlich, das Wissen um mögliche systembedingte Ursachen unfreundlichen Verhaltens im Hinterkopf zu behalten.

\subsection{Die Rolle von Bildern in der Online- Kommunikation}

Durch die zunehmend größeren Kapazitäten für die Datenübertragung ist Online-Kommunikation heute nicht mehr zwingend auf die Schriftlichkeit reduziert. Sie wird durch komplexere Kanäle (Ton, Bild, Video) ergänzt und eröffnet dadurch ein breiteres Spektrum an Ausdrucksmöglichkeiten. Es ist anzunehmen, dass sich dieser Trend weiter verstärken wird und in vielen Bereichen - die heute rein textbasiert sind - immer häufiger ergänzend auf Bilder gesetzt wird. Das Bedürfnis zu dieser Erweiterung ist in den unterschiedlichen kommunikativen Möglichkeiten von Text und Bild begründet.

\subsubsection{Wie sich Bild und Text ergänzen}

Um die spezifische Wirkung von Bild und Text zu verstehen, kann man auf eine weitere Kommunikationsregel von Watzlawick et al. $(1969,2000)$ zurückgreifen.

\section{Menschliche Kommunikation bedient sich digitaler und analoger Modalitäten (4. Axiom)}

Das bedeutet: Bild und Text haben ein unterschiedliches Potenzial und unterstützen jeweils das Verständnis verschiedener Aspekte einer Botschaft.
Text ist präzise beschreibend, Bilder übermitteln Emotionen und Zusammenhänge 
Bildbeschaffung verursacht Kosten
Digitale Kommunikation (Text). »Digital« wird hier nicht im Sinne von »computerunterstützt« verwendet, sondern steht für alle Arten von zeichenbasierter, abstrakter Kommunikation, für Konventionen, die festlegen, wie ein Inhalt kodiert wird (Zahlen, Buchstaben, Kodierungssysteme). Die festgelegten Zeichen haben keine äußere Ähnlichkeit mit dem Inhalt, den sie beschreiben. Beispiel: Ka z z ist eine Reihenfolge von Zeichen, die ein Tier definiert. Digitale Kommunikation kann Inhalte exakt und logisch beschreiben.

Analoge Kommunikation (Bild). »Analog « heißt, dass zwischen der Darstellung eines Gegenstandes und ihm selbst eine Ähnlichkeit, eben eine Analogie besteht. Diese Kommunikationsform ist viel älter als die digitale, und wir teilen sie mit den Tieren, die ebenfalls und ausschließlich analog kommunizieren. Analoge Kommunikation ermöglicht die treffsichere Deutung von emotionalen Zusammenhängen und Beziehungsaussagen. Beispiel: Unaufrichtigkeit wird in der Körperhaltung einer Person relativ rasch festgestellt, in einem Text hingegen ist das sehr schwierig.

Die digitale Kommunikation (Text) gibt also Auskunft über den Inhalt einer Nachricht, der Beziehungsaspekt wird analog vermittelt (Bild).

\section{(1) Rein textbasierte Kommunikation hat den Nachteil, dass emotionale Aspekte zu kurz kommen, die bildbasierte Kommunikation wiede- rum ist nicht exakt genug, um komplexe Sachverhalte treffend zu beschreiben.}

Ergänzend zu Watzlawick kann man sagen, dass die bildbasierte Kommunikation auch das schnelle Erfassen eines Zustandes, eines Sachverhalts in der Einbettung seines Umfelds ermöglicht - wenn auch vorwiegend intuitiv und ohne den Detaillierungsgrad eines Textes. Bilder können somit eine ähnliche Funktion übernehmen wie Inhaltsverzeichnisse in einem Text - als konzeptionelle »Oberbegriffe« -, die die Einordnung von Informationen in ein größeres Ganzes erleichtern (zum menschlichen Informationsverarbeitungsprozess $>$ Kap. 5.4.3).

Bilder können Text im Internet nicht ersetzen, sie können aber die Kommunikation um wichtige Aspekte ergänzen. Die analoge (Bild-) Kommunikation entspricht dem menschlichen Grundbedürfnis, Inhalte emotional einordnen zu können und auf ihre Glaubwürdigkeit zu überprüfen. Sie kann außerdem die konzeptgesteuerte Verarbeitung von Informationen unterstützen.

Online-Newsanbieter kommen diesem Bedürfnis vermehrt entgegen, indem sie $\mathrm{zu}$ wichtigen Themen »Bildstrecken « anbieten. Auch für die Online-Vertrauensbildung ( $\triangleright$ Kap. 8.2.2) haben Bilder eine wichtige Bedeutung, z. B. die Präsentation von Fotos der Unternehmensleitung auf der Website, um emotionale Informationen zu übermitteln.

Die Verwendung von Bildern im Internet hat allerdings auch gewisse Nachteile, sie ist z. B. oft mit zusätzlichen Kosten verbunden (Bildbeschaffung). Je nach Kommunikationsaufgabe machen Bilder die Informationsübermittlung unnötig kompliziert ( $\triangleright$ Kap.3.1.2, Medienwahl und Kommunikationsaufgabe). Suchmaschinen können rein bildbasierte Informationen - zumindest bisher - nur schwer auffinden ( Kap. 8.4.11, Such- 
maschinenoptimierung). Außerdem sind wichtige Online-Kommunikationskanäle nach wie vor mehrheitlich textbasiert, z. B. der E-Mail-Verkehr, Newsforen oder Blogs. Es ist darum trotz der Tendenz zur vermehrten Verwendung von Bildern wichtig, die Eigenschaften und Folgen von rein textbasierter Kommunikation zu kennen.

\subsection{Das digitale Datenformat und seine psychologischen Folgen}

Die digitalisierte Informationsübermittlung ist die augenfälligste Eigenschaft der Online-Kommunikation. Im Unterschied zu den Kanal- und Filtertheorien steht hier nicht die Nachricht oder der Sender im Zentrum, sondern das technische Datenformat. Die Digitalisierung hat eine ungemein starke Effizienzzunahme bei der Informationsübertragung zur Folge. Inhalte können fast beliebig schnell und sehr kostengünstig an jeden gewünschten Ort geschickt, vervielfältigt, verändert und archiviert werden. Dies ist auch eine wichtige Ursache für die viel zitierte Informationsflut.

Das digitale Datenformat hat ambivalente Auswirkungen, das heißt - je nach Nutzungsart - positive und negative Folgen. Dies gilt gerade auch für psychologische Prozesse und das menschliche Verhalten als Reaktion auf die neue Art der Informationsaufbereitung. Die nun folgende Beschreibung der verschiedenen Folgen digitaler Kommunikation basiert auf der Kategorisierung von Döring (2003, S. 157ff), mit eigenen Ergänzungen.

\subsubsection{Entlastung und Überlastung}

Suchmaschinen und andere Strukturierungshilfen wie Taxonomien, Online-Verzeichnisse oder Newsreader spielen im Internet und auch in unternehmensinternen Netzwerken mehr und mehr eine zentrale Rolle. Sie ermöglichen es, Informationen nach den eigenen Bedürfnissen zu gliedern, zu kanalisieren und somit überhaupt erst nutzbar zu machen ( $\downarrow$ Kap. 13.4).

Dies ist dann auch die erste ambivalente Folge der Digitalisierung: Wer neue Medien nutzt, hat einerseits fast unbeschränkte Möglichkeiten zur Gewinnung von Daten, die ihm für verschiedene Zwecke nützlich sind. Andererseits muss er sich dafür durch eine Unzahl von Informationen hindurchkämpfen und wird permanent dazu gezwungen, sich für die einen Informationswege zu entscheiden und andere auszulassen ( $\mathrm{\bullet}$ Abb. 2.2). Dadurch entstehen Überlastungsgefühle.

Online-Foren oder andere Netzwerke sind gute Beispiele, um den entlastenden Effekt aufzuzeigen. Angenommen, jemand hat eine seltene Krankheit, die in seinem eigenen Land vielleicht nur fünfmal vorkommt. Auch wenn er bis jetzt noch nie jemanden kennen gelernt hat, der in der gleichen Situation ist, findet er im Internet im $\mathrm{Nu}$ mindestens eine Gruppe von ebenfalls Betroffenen, mit der er sich austauschen kann und die ihm gezielte Unterstützung gibt. Oder er kann selbst eine Gruppe gründen und ist dank der Suchmaschinen für andere schnell auffindbar.
Die Folgen der Digitalisierung sind ambivalent

Die Strukturierung von Informationen macht diese erst nutzbar 
- Abb. 2.2. Unterwegs im digitalen Dschungel

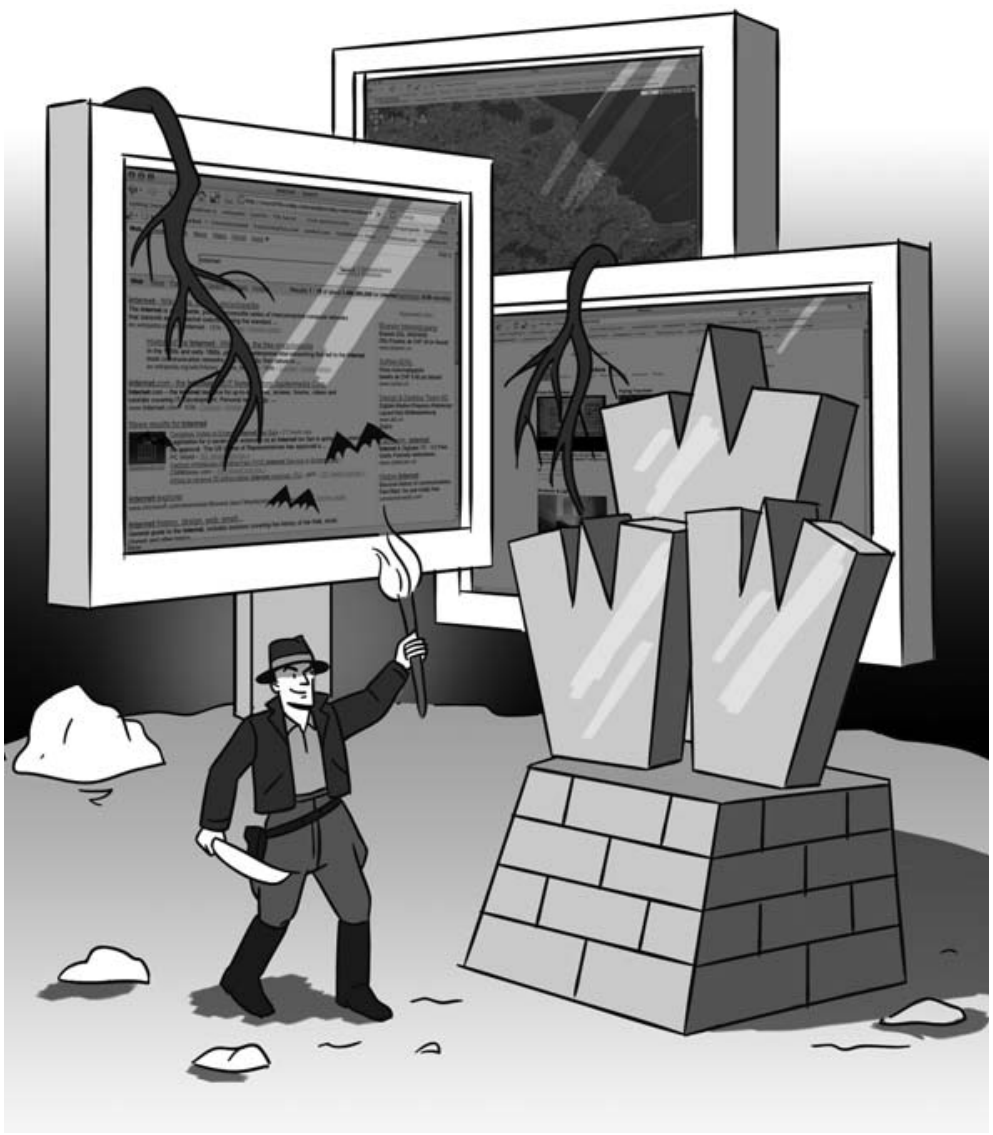

Diese schnelle und unkomplizierte Vernetzung macht einen großen Teil der Attraktivität des Internets aus und wird durch das digitale Datenformat überhaupt erst möglich. Dass die Zahl von »Social Networks« und Interessengruppen im Internet rasant zunimmt, ist ein Beweis dafür, dass trotz der Gefahr drohender Informations-Überlastung viele Menschen die Möglichkeiten zur Entlastung gut zu nutzen wissen.

\subsubsection{Kontrolle und Kontrollverlust}

Die Tatsache, dass digitale Informationen einfacher zu kontrollieren sind, ist ebenfalls ambivalent. Drei Ebenen sind dabei relevant (Döring, 2003):

- Die persönliche Möglichkeit zur Kontrolle von Informationen,

- die persönliche Notwendigkeit, Kontrolle über Informationen zu erreichen und zu erhalten sowie

- die Möglichkeit und das dadurch implizierte Risiko, dass andere Personen über meine Informationen Kontrolle ausüben. 
Im Folgenden werden diese Aspekte der Anschaulichkeit halber anhand der persönlichen Ebene illustriert. Sie gelten aber ebenso für Unternehmen, denn diese haben im digitalen Zeitalter z. B. neue Archivierungsmöglichkeiten und -pflichten für geschäftsrelevante Informationen, und sie müssen ihre Mitarbeitenden für die Gefahren der Kontrollierbarkeit digitaler Informationen sensibilisieren.

(? Seit der gesamte E-Mail-Verkehr eines Unternehmens als Beweismaterial in Gerichtsprozessen verwendet werden kann, ist die Diskrepanz zwischen der schnellen Erstellung einer E-Mail und ihrer beinahe unbeschränkten Haltbarkeit besonders fatal.

\section{Persönliche Möglichkeit zur Kontrolle}

Aus der psychologischen Forschung weiß man, wie wichtig es für den Menschen ist, Kontrolle ausüben zu können. Dies nicht negativ gemeint, im Sinne der Machtergreifung über einen anderen Menschen, sondern als Beherrschung der alltäglichen Pflichten und Herausforderungen. Das Gefühl, eine Aufgabe meistern zu können, stärkt das Selbstwertgefühl, vermittelt Befriedigung und gibt den Mut, neue Dinge in Angriff zu nehmen. Der Verlust von Kontrolle führt zu Stress und Hilflosigkeitsgefühlen und je nachdem zur Vermeidung der Tätigkeiten, die zum Kontrollverlust geführt haben ( $\triangleright$ Kap. 13).

Digitale Informationen erlauben durch die einfache Suchbarkeit, Beschlagwortung und Archivierung ein hohes Maß an Kontrolle. Die Tendenz, digitale Informationen länger aufzubewahren als physische, wird zusätzlich durch die hohe Speicherkapazität von Festplatten und anderen Speichermedien begünstigt. Wenn man z. B. vor drei Jahren eine E-Mail an eine Person geschrieben hat, von der man nur noch weiß, dass sie "Rita" heißt, kann man mittels Suchfunktion in der Mailbox binnen kurzer Zeit genau diese E-Mail finden (vorausgesetzt, man hat das E-Mail-Archiv nie gelöscht). Dies kann eine große Erleichterung bedeuten, weil man Daten wieder finden oder Sachverhalte rekonstruieren kann, die früher verloren gegangen wären, weil der Aufwand zur systematischen Aufbewahrung physischer Briefe viel größer ist.

Anbieter von Suchmaschinen ermöglichen heute, die gesamte Arbeitsumgebung eines Unternehmens nach Stichworten abzusuchen. Wo Informationen strukturiert abgelegt sind, bedeutet dies Effizienzgewinn und Arbeitserleichterung.

\section{Beispiel}

Ungeliebter Kontrollversuch:

automatische E-Mail-Empfangsbestätigung

Die meisten E-Mail-Dienste ermöglichen es, den Empfang einer E-Mail bei der Zielperson automatisch bestätigen zu lassen. Diese Option sollte man nur sehr sparsam einsetzen und keinesfalls als permanente Einstel-
Kontrolle vermeidet Stress 
Empfangsbestätigung als Misstrauensvotum
Auch Unbefugte können über digitale Informationen einfacher Kontrolle erlangen lung der Mailbox. Sie kann beim Empfänger leicht ein unerwünschtes Bild entstehen lassen: nämlich dass der Sender Kontrolle über ihn ausüben will. Die Asynchronizität gibt einem die freie Wahl, eine E-Mail genau dann zu lesen, wann man dazu Lust hat. Indem man als Sender eine Empfangsbestätigung verlangt, tastet man diese freie Wahlmöglichkeit an und zwingt das Gegenüber, etwas über seine Lesegewohnheiten zu verraten.

Viele Nutzer haben die Empfangsbestätigung optional gesetzt, das heißt, sie entscheiden sowieso bei jeder E-Mail frei, ob sie jemandem diesen Einblick ins Leseverhalten gewähren. Daraus entstehen für den Sender gleich zwei negative Folgen:

- Einerseits hat man beim Empfänger einen schlechten Eindruck als Kontrollfanatiker hinterlassen,

- andererseits fehlt oft trotzdem die Sicherheit, dass die E-Mail gelesen wurde.

Besser ist es, inhaltlich statt mit technischen Empfangsbestätigungen zu arbeiten: einen klaren Termin für eine zu erledigende Aufgabe setzen und in der Formulierung einer Anfrage exakt und verbindlich sein. So kann man rechtzeitig nachfragen, wenn man keine Reaktion erhält. Man kann auch zum Telefon greifen und so gegebenenfalls Verständnisprobleme beseitigen.

Vermeiden sollte man Empfangsbestätigungen v. a. für die Kommunikation mit einer tieferen Hierarchiestufe - das kann als Misstrauen oder autoritärer Führungsstil ausgelegt werden. Im Normalfall werden EMails eines Vorgesetzten sowieso rasch bearbeitet - aus Respekt vor der Führungsperson.

\section{Persönliche Notwendigkeit zur Kontrolle}

Kontrolle über seinen »Informationshaushalt" $\mathrm{zu}$ halten, bedingt viel Selbstdisziplin. Das stetige Abwägen, welche Informationen man behalten soll und welche gelöscht werden können, ist zeitintensiv und auch inhaltlich anspruchsvoll. Tendenziell neigt man darum dazu, eher zu viele Informationen aufzubewahren - zusätzlich begünstigt durch den Umstand, dass die digitale Ablage weder viel Platz noch viel Geld kostet. Mittelfristig ist es aber nicht unbedingt vorteilhaft, Informationen ungefiltert liegen zu lassen, weil die nachträgliche Sichtung von Daten viel aufwändiger ist.

Auch die Verwaltung der eigenen Mailbox stellt uns permanent vor das Problem, dass wir trotz Zeitknappheit die relevanten Informationen nicht verpassen dürfen.

Dies bedeutet, dass man am digitalen Arbeitsplatz permanent gefordert ist Kontrolle auszuüben, um den langfristigen Kontrollverlust $\mathrm{zu}$ vermeiden. Daraus resultierende Stressgefühle können die Arbeitsleistung beeinträchtigen. 


\section{Beispiel}

\section{Digitale Entscheidungssituationen}

»Allein schon die Entscheidung zu treffen, ob du eine E-Mail löschst oder nicht, ist eine größere Sache. Wenn du dich entscheidest, sie nicht zu löschen, dann muss sie ja archiviert werden, und man stelle sich vor, was das für eine riesige Datenmenge ist, wenn alle Mitarbeiter pro Tag 50-100 Mails kriegen. Die Frage ist dann auch wieder, hebt man die Mails auf, um irgendwas für später einmal zu dokumentieren. Aber das ist dann wieder wie ein Vertrauensbruch. Man hat wahrscheinlich eine große Angst, dass Informationen verloren gehen und man etwas nicht mehr nachweisen kann. Man fühlt sich so in einer Pseudosicherheit, man hat ja alles da. Dabei gibt es kein vernünftiges System, die Information wieder rauszusuchen. Es ist jedes Mal eine Aktion, im Archiv etwas zu finden.« W, 43, Mittleres Management, global tätiges Pharmaunternehmen

\section{Kontrolle anderer über meine Informationen}

Ein Aspekt von großer Tragweite ist die Kontrollierbarkeit von Informationen durch andere. Der »gläserne Mensch « im Internet ist eine Realität geworden, der wir uns nicht entziehen können ( $\checkmark$ Kap. 14.6). Aber auch für Unternehmen ist das Thema brisant, denn vertrauliche Informationen können nicht mehr so einfach gegen Zugriff geschützt werden wie früher, und eine einzige Person als Sicherheitslücke reicht aus, um Indiskretionen in Windeseile weltweit $\mathrm{zu}$ verbreiten und dadurch unkontrollierbar $\mathrm{zu}$ machen. Die Digitalisierung bewirkt einen nie da gewesenen Multiplikatoreffekt. Mehr dazu $\triangleright$ Kap. 11 (Online-PR).

\subsubsection{Entscheidungsmöglichkeiten und Entscheidungszwang}

Die Informationsaufbereitung im Internet in Form von Hyperlinks ermöglicht neue Arten von assoziativer Recherche. Aber auch wenn Informationen leicht zugänglich sind, ist es nicht immer einfach, gute Quellen $\mathrm{zu}$ finden und sie von schlechten zu unterscheiden. Die erfolgreiche Selektionsarbeit bedingt Kenntnisse über Suchstrategien und z. B. auch den Aufbau von Internet-Adressen. Hilfestellungen zu diesem Thema sind in - Kap. 13.3.4 zu finden. Eine Übersicht über psychologische Forschung im Zusammenhang mit Informationssuche und Entscheidungsprozessen bei der Informationsauswahl findet sich bei Wirschum (2006).

\subsubsection{Erweiterter Teilnehmerkreis}

Wie oben bereits angedeutet, können Informationen praktisch ohne Mehraufwand an beliebig viele Personen gesendet werden. Mit jedem zusätzlichen Empfänger wächst aber das Risiko von Komplikationen. Im harmlo-

Je mehr Teilnehmer, desto größer das Komplikationsrisiko 
Aufgaben müssen permanent neu priorisiert werden sesten Fall ist das ein Beitrag zur Überflutung einer fremden Mailbox. Es können aber auch Missverständnisse, Unsicherheiten und Ärger entstehen, und dies proportional zur Anzahl der Empfänger.

\section{(? Die problemlose Erweiterung des Teilnehmerkreises ist ein Grund dafür, dass man wirklich vertrauliche Dinge nicht via E-Mail übermitteln sollte. Sogar eine verschlüsselte E-Mail kann von einem gedankenlosen Empfänger an Unbefugte weiterge- sendet werden.}

Dies zwingt den verantwortungsbewussten Sender, sich vor dem Abschicken immer Gedanken darüber zu machen:

- Wen die Mitteilung tatsächlich etwas angeht,

- ob er mit einer Veröffentlichung der Mitteilung in einer Tageszeitung leben könnte,

- ob er abschätzen kann, welche unterschiedlichen Gefühle, Stimmungen und Reaktionen seine Nachricht bei den Empfängern auslöst und ob er mit diesen angemessen umgehen kann.

Die Verwendung von Cc (Kopie) und Bcc (Blindkopie) bei E-Mails birgt nicht selten ein zusätzliches Konfliktpotenzial, da die Rollen der KopieEmpfänger nicht immer klar definiert sind. Hier kumuliert sich die einfache Erweiterung des Teilnehmerkreises mit der Schwierigkeit, eine kanalreduzierte Nachricht zu interpretieren ( Kap. 2.2.1). Mehr zur richtigen Anwendung von $\mathrm{Cc}$ und Bcc $\triangleright$ Kap. 7.3.

\subsubsection{Gesteigerte Transport- und Bearbeitungsgeschwindigkeit}

Arbeitsaufträge können mittels E-Mail innerhalb von Sekunden übermittelt werden. Dadurch fallen räumliche und zeitliche Filter weg, die bis jetzt den Empfang neuer Aufträge strukturiert und getaktet haben. Räumliche Filter bedeuteten früher höhere Kosten der Kommunikation. Heute findet dank der praktisch kostenlosen digitalen Informationsübermittlung eine Beschleunigung des Transports statt, die auch nach erhöhtem Bearbeitungstempo verlangt. Die Mailbox zwingt heute zum permanenten Sichten und Priorisieren von sich stetig verändernden Aufgaben. Dadurch können Projekte schneller vorangetrieben werden. Aber auch der Stress für die Empfänger von Aufgaben nimmt im gleichen Maß zu ( $\triangleright$ Kap. 13, digitaler Stress am Arbeitsplatz).

\subsubsection{Variable Empfängerzahl bei gleich bleibenden Bearbeitungsressourcen}

Bei digitaler Kommunikation nimmt nicht nur die Geschwindigkeit der Aufgabenübermittlung zu. 


\section{(D) Durch die Erweiterung des Teilnehmerkreises kann das Arbeits- volumen rasch überproportional ansteigen. Dessen muss man sich besonders dann bewusst sein, wenn man über wenig variable Res- sourcen zur Betreuung von interaktiven Online-Angeboten verfügt.}

Zum Beispiel ist es einer Einzelperson ein Leichtes, ein Dienstleistungsangebot an eine Gruppe von 200 Personen zu senden. Sie muss nachher aber grundsätzlich die Anfragen all dieser 200 Leute bewältigen können, und zusätzlich solche von Dritten, denen das Angebot weiterempfohlen wurde. Auch der Unterhalt von Blogs oder anderen stark interaktiven Elementen einer Website kann aus diesem Grund eine unerwartet ressourcenintensive Aufgabe werden. Dieser Aspekt muss bei der Gestaltung von Online-Dialogangeboten unbedingt berücksichtigt werden ( Kap. 12.5, das richtige Maß an Interaktivität).

\subsubsection{Die Sorgfaltspflicht bei Online-Texten}

Die textbasierte Online-Kommunikation verlangt von den Benutzern eine hohe Kompetenz für die schriftliche Ausdrucksfähigkeit. Diese ist nicht immer gegeben. Trotzdem hat sich E-Mail in der geschäftlichen Kommunikation als meistverwendetes Medium durchgesetzt. Dies - kombiniert mit einem erhöhten Zeitdruck und der Schnelligkeit der digitalen Übermittlung - führt dazu, dass man häufig Online-Texten begegnet, die schlecht geschrieben sind, Tippfehler und grammatikalische Schwächen enthalten. Dies muss nicht gezwungenermaßen bedeuten, dass eine »Verarmung « unserer Sprachkultur stattgefunden hat. Es ist vielmehr so, dass heute z. B. auch technisch ausgerichtete Informatiker ohne ausgeprägtes Sprachflair um das schriftliche Kommunizieren nicht mehr herumkommen. So gesehen, kann die OnlineSchreibkultur auch als Chance gesehen werden, weil Leute zum Schreiben "gezwungen« werden, die das sonst nicht oder seltener tun würden.

Trotzdem hat die Nachlässigkeit im Schreibstil auch ihre Tücken, gerade in der Kommunikation mit Kunden. Bei Briefen, die gedruckt verschickt werden, stellt der Ausdruck auf Papier einen Filter dar, eine natürliche Barriere, die verhindert, dass der Text allzu schnell geschrieben und nicht auf Fehler überprüft wird. Auch die strengeren Gestaltungsvorschriften (Briefkopf, Corporate Identity) mahnen den Sender zu erhöhter Sorgfalt. Diese natürlichen Filter fallen bei der E-Mail-Kommunikation weg. Trotzdem sind viele Empfänger nicht bereit, über Tippfehler und mangelhafte Formulierungen in schlampig geschriebenen E-Mails hinwegzusehen. Die fehlende Sorgfalt wird als mangelnde Wertschätzung empfunden, im schlimmsten Fall (wenn es keine äußeren Möglichkeiten gibt, das Fehlverhalten zu entschuldigen) wird der Sender als unseriös betrachtet oder nicht ernst genommen. Auch Spam- oder Phishing-E-Mails erkennt man oft daran, dass sie Tippfehler und falsche Formulierungen enthalten.

Es ist äußerst wichtig, gerade in der offiziellen Kommunikation mit Kunden, eine E-Mail, einen Newsletter oder eine Website mit der gleichen Sorgfalt zu gestalten wie einen gedruckten Brief oder eine Print-Broschüre.
Interaktive Angebote wie Blogs oder Foren sind ressourcenintensiv

Fehlende Sorgfalt wird als mangelnde Wertschätzung empfunden 
Eine Einzelperson kann ein Online-Erdbeben auslösen
Dies gilt grundsätzlich auch im persönlichen E-Mail-Kontakt mit Leuten, die man noch nicht oder nicht gut kennt. Erst bei besserer Bekanntschaft kann man sich erlauben, einen unkomplizierteren Schreibstil anzuschlagen. Zur Textgestaltung in E-Mails — Kap. 7.3.2.

\subsection{In der Praxis: Wie sich die Eigenschaften der Online-Medien auswirken}

\subsubsection{Hierarchien in der Online-Kommunikation}

Seit dem ersten Internet-Hype in den 90er-Jahren wurde immer wieder angeführt, dass das Internet die bisherigen Hierarchien aufbrechen und Machtstrukturen auf den Kopf stellen werde. Ganz so radikal, wie man sich das damals vorstellte, hat sich diese Prophezeiung nicht bewahrheitet. Nach wie vor ist nicht jeder Mitarbeiter mitspracheberechtigt bei den Entscheiden der Geschäftsleitung, und er erhält auch weiterhin nicht alle Informationen, die ein Chef zur Verfügung hat. Aber in gewisser Weise ist es online tatsächlich einfacher geworden, sich als Angehöriger einer Minderheit oder einer hierarchisch tiefer gestellten Gruppe Gehör zu verschaffen. Dies hat einerseits mit der Digitalisierung zu tun ( $\checkmark$ Kap. 2.5), die die technischen Grundlagen für die Nivellierung der Online-Kontakte schafft. So sind z. B. oft alle E-MailAdressen eines Unternehmens gleich aufgebaut und jeder Mitarbeiter weiß, wie er den CEO direkt ansprechen kann. Andererseits können auch die oben genannten Filter-Effekte ( $\triangleright$ Kap. 2.3) mitspielen: Ich vertraue mich meinem CEO eher an, wenn ich ihn nicht sehe und er mich dann auch nicht durch seine Präsenz einschüchtert (was sonst vielleicht der Fall wäre).

\section{(. Den wichtigsten Einfluss auf Hierarchien haben die neuen Medien durch die unkontrollierbare Verbreitung von Informationen und die Schwierigkeit, unerwünschte Inhalte zu zensieren.}

Das Internet ist für jedermann sehr leicht zugänglich, was dazu führt, dass die Kritik einer lokalen Einzelperson oder Gruppe gegenüber einer global tätigen Firma potentiell ein Erdbeben auslösen kann. Da sind Manager heute gefordert, offensiver zu kommunizieren und - wenn die Kritik berechtigt ist - mit solchen Stakeholdern aktiv in Dialog zu treten. Dieser Zwang bestand früher in der Form weniger, weil es für Anspruchsgruppen schwieriger war, sich mit Gleichgesinnten zu organisieren und öffentlich wahrgenommen zu werden. Siehe dazu auch die Ausführungen in $\$ Kap. 4 (Web 2.0) und $\triangleright$ Kap. 11 (Online-PR).

\subsubsection{Wo die neuen Medien traditionelle Hierarchien in Frage stellen}

Führungskräfte sind heute mit veränderten hierarchischen Strukturen durch das Internet konfrontiert. Dies zeigt sich in unterschiedlicher Weise im Unternehmensalltag. 


\section{Checkliste: Neue Medien und traditionelle Hierarchien}

\section{Auf individueller Ebene}

- Der Chef erhält persönliche Mails von einfachen Angestellten zu möglicherweise für inn irrelevanten Themen (Regel).

- Der Chef erhält persönliche Mails von einfachen Angestellten zu höchst relevanten Themen, von denen er sonst nie erfahren hätte (Ausnahme, weil das auch per E-Mail sehr viel Zivilcourage voraussetzt).

- Der Chef erhält Spam und Werbemails in seine persönliche Mailbox.

- Der Chef wird in E-Mails nicht seinem Status gemäß angesprochen (sondern salopp oder schnoddrig).

- Der Chef wird ungefragt als Druckmittel gegenüber Dritten eingesetzt (indem man inn ins Cc: einer Mail setzt).

- Der Chef wird innerhalb des E-Mail-Adressverteilers seiner hierarchischen Höherstellung beraubt (im Adressfeld ist sein Name gleichrangig mit denjenigen der einfachen Mitarbeitern aufgeführt).

- Der CEO wird ungefragt von Kunden oder Verkäufern angegangen, auch wenn tiefer gestellte Vorgesetzte die richtigen Ansprechpersonen wären, und muss darauf reagieren.

\section{Auf Firmenebene}

- Die Firma wird von einer hierarchisch »unbedeutenden« Einzelperson in einem Internet-Forum kritisiert, was einen Medienrummel auslöst und die Firma zum Handeln zwingt.

- Die Firma unterhält einen eigenen Corporate Blog und wird mit bösen Kommentaren von Dritten öffentlich bloßgestellt.

- In einem Forum im Firmen-Intranet kommt Kritik am neuen Lohnsystem der Firma auf. Immer mehr Leute schließen sich den Unzufriedenen an und die Stimmung unter den Angestellten verschlechtert sich dramatisch.
Attacken auf die Hierarchie sind systemimmanent
Es kann für einen Vorgesetzten entlastend sein, zu merken, dass die »Attacken" auf seine hierarchische Stellung ein Phänomen sind, das mit der Nutzung der neuen Medien im Unternehmensalltag zu tun hat und nicht mit seiner Person. Gleichzeitig fordert es ihn aber zu einem neuen Umgang mit seinen Angestellten heraus: Er soll anzeigen, wo eine Grenzüberschreitung nicht akzeptabel ist, aber auch partnerschaftlich mit seinen Untergebenen in Austausch treten und ihre Anliegen ernst nehmen. Beziehen sich die Angriffe auf das Unternehmen, sind ebenfalls neue Kommunikationskompetenzen und ein neuer Umgangston gefragt. Mehr dazu $\triangleright$ Kap. 11. 


\title{
"Die menschliche DNA erlaubt uns nicht, uns vom hierarchischen Denken zu entfernen «
}

\author{
Interview mit Fred Kindle, zum Zeitpunkt des Interviews CEO ABB Group
}

\section{Herr Kindle, welche Rolle spielen E-Mail und Internet im Unternehmensalltag von ABB?}

Wir sind bei $\mathrm{ABB}$ weltweit vernetzt und synchronisiert, E-Mails bleiben nicht lange liegen, sie sind zentraler als der Schriftverkehr. Alle Mitarbeiter haben einen Laptop oder einen Personal Organiser, ähnlich dem Blackberry. Innerhalb unserer Gebäude kann man mit Wireless LAN überall ins Netz und seine E-Mails abrufen. Dies ist sehr bequem und nützlich für ein globales Unternehmen.

Diese Vernetzung hat aber auch negative Konsequenzen: Unsere Leute sind teilweise fast süchtig danach. Es kommt sogar in Verwaltungsrats- und Geschäftsleitungssitzungen vor, dass Sitzungsteilnehmer, während ein anderer spricht, ihre E-Mails herunterladen und lesen. Das geht natürlich zu weit und ist der internen Effizienz abträglich. An meinen Meetings habe ich darum kurzerhand Laptops und Organisers verboten, außer wenn einer selbst eine Präsentation hält oder Protokoll führen muss.

Ein weiterer Nachteil von E-Mail hängt mit der Auswahl der Adressaten zusammen. Ich stelle fest, dass viele E-Mail-Sender nicht rigoros genug sind bei der Wahl ihrer Ansprechpartner. Sie senden ihre Mails an zu viele Empfänger, dadurch ergibt sich ein dramatisches Wachstum des Adressatenkreises und der Anzahl E-Mails in der In-Box. Auch Attachments werden oft zu großzügig hinzugefügt, so dass man mit der Informationsmenge gar nicht mehr wirklich etwas anfangen kann.

\section{Gibt es etwas, worauf Sie persönlich besonders Acht geben, wenn Sie eine E-Mail senden?} Ich achte immer darauf, dass ich meine E-Mails möglichst präzise formuliere. Gerade für einen CEO ist das sehr wichtig, damit ich Missverständnisse verhindern kann. Wenn ich mit jemandem spreche, frage ich ja oft nach: Verstehen Sie, was ich meine? Und wenn der andere es nicht verstanden hat, kann ich noch mal mit anderen Worten das Gleiche sagen. Bei E-Mails geht das eben nicht, darum muss man sich umso mehr Mühe geben, was und wie man etwas sagt. Auch den Adressatenkreis wähle ich sehr bewusst. Nicht maximieren, sondern beschränken.

\section{Die Internet-Gründergeneration hat in den frühen 90er-Jahren prophezeit, das Netz werde alle Hierarchien und Standesunterschiede aufheben und die totale Demokratie herbeiführen. Wie sehen Sie das aus heutiger Sicht?}

Trotz vieler moderner Kommentare zu Netzwerken und nichthierarchischen Arbeitsgruppen glaube ich nicht, dass die menschliche DNA uns erlaubt, uns vom hierarchischen Denken und Handeln zu entfernen. Der Mensch ist ein Rudeltier wie der Hund, er ist geprägt von der Hierarchie, er sucht sie auch und scheint manchmal verloren ohne eine hierarchische Einbindung. Die Hierarchie ist ein sinnvolles Mittel, um eine Gruppe zu koordinieren, so dass sie gewisse Ziele erreichen kann. Selbstverständlich gibt es bei der Anwendung von hierarchischen Strukturen oft Probleme mit Wissens- und Machtmissbrauch. Im geschäftlichen Alltag geht es genau darum, diesen zu vermeiden und die Hierarchie »Nutzen stiftend « und transparent einzusetzen.

Ich glaube darum nicht, dass das Internet Hierarchien aufheben kann. Aber es entsteht dank dem Internet eine hohe Transparenz. Für einzelne Personen wird es immer schwieriger, eine hi- 
erarchische Position auszunutzen, ohne entdeckt zu werden. Missbräuche werden schneller bekannt, Manipulation gelingt nicht mehr so einfach, die Kontrolle von geheimen Informationen wird schwieriger.

Interessant ist auch der Umstand, dass nicht mehr nur große Systeme wie Medienverlage oder Unternehmen den Meinungsbildungsprozess prägen, sondern im Extremfall eine einzelne Person in einem Blog so einen Prozess auslösen kann. Diese zunehmende Transparenz im Netz ist sicher Demokratie fördernd. Die Gesellschaft wird in ihrem Meinungsbildungsprozess noch pluralistischer. Ich glaube aber, dass die Hierarchien an sich weiter bestehen bleiben.

Leider gibt es auch einen unerwünschten Nebeneffekt, den ich »Exzess an Transparenz« nenne: Durch die globale Real-Time-Zugänglichkeit von Informationen haben wir keine »Gnadenfrist «mehr, auf überraschende Entwicklungen zu reagieren. Das SARS-Phänomen z. B. hätte früher keinen Kollaps von Fluggesellschaften und Reisevermittlern ausgelöst. Wir hätten gar nicht rasch genug gemerkt, dass eine potenzielle Gesundheitsgefährdung besteht, bevor sie bereits wieder verschwunden wäre. Die Schnelligkeit der weltweiten Informationsübermittlung, kombiniert mit der menschlichen Schwäche, alles zu emotionalisieren und zu dramatisieren, führt zu einer Übersteigerung, die reale Auswirkungen hat. Ich merke das auch bei Neuigkeiten über unser Unternehmen. Da kann es vorkommen, dass ich ein Interview gebe, ein Journalist gewisse Inhalte etwas verkürzt berichtet, die internationalen Agenturen verkürzen es noch mal zusätzlich, was zu einer überspitzten Darstellung auf dem Börsen-Newsticker führt - und schon fällt unser Aktienkurs um zwei Prozent, ohne dass es irgend einen realen Grund dafür gäbe. Erst wenn die Analysten dann das ursprüngliche Interview lesen, beruhigt sich die Sache wieder.

\section{Was tun Sie, um möglichen Schwierigkeiten oder Nachteilen entgegenzuwirken, die Ihnen aus Ihrer hierarchischen Stufe entstehen?}

Für die Unternehmensspitze ist ein wichtiges Problem, dass wir Feedback von der Unternehmensbasis oft nur gefiltert, nicht ehrlich oder gar nicht erhalten. Die Offenheit nach oben war immer schon durch hierarchische Ängste eingeschränkt. Ich muss also dafür sorgen, dass ich trotzdem wichtige Informationen aus dem geschäftlichen Alltag erhalte und mir ein Bild über die aktuelle Situation machen kann. Dazu gibt es natürlich verschiedene Mittel. Unter anderem lade ich unsere Mitarbeiter dazu ein, mir aktiv ihre Meinung per E-Mail zu äußern. Meine CEOBriefe, die per E-Mail verteilt werden, enthalten am Schluss immer einen integrierten FeedbackButton. Die Mitarbeiter können darin ihre Meinung kundtun, die wird dann an Corporate Communications gesendet und von dort gesammelt an mich weitergegeben.

Wir merken, dass dieser Feedback-Button v. a. in Krisensituationen sehr rege genutzt wird. Wenn sich die Situation stabilisiert hat oder die Relevanz des angesprochenen Themas etwas tiefer ist, nimmt das Feedback über diesen Kanal automatisch wieder ab. Grundsätzlich haben wir eine relativ gut ausgeprägte Feedbackkultur, das hat auch mit vergangenen Erfahrungen unseres Unternehmens zu tun.

\section{Denken Sie, dass die elektronischen Medien den Informationsfluss zwischen Ihnen und Ihren Angestellten verbessern?}

Unser Intranet ist für die Kommunikation zentral. Wir schalten täglich News auf, und es gibt Seiten in unserem Intranet, auf denen die Leute ihre Anliegen über alle hierarchischen Stufen 
hinweg mitteilen können. Dies gilt insbesondere für das »Whistleblowing« betreffend ethischem Geschäftsgebaren, wo wir ganz klar definierte Prozesse aufgesetzt haben.

\section{Können Sie ein Erlebnis erzählen, wo Ihnen eine Person aus einer tiefen Hierarchiestufe ungefragt eine E-Mail gesendet hat? Wie haben Sie darauf reagiert?}

Ich erhalte etwa alle zwei Wochen solche E-Mails von Mitarbeitenden. Gerade gestern beklagte sich einer über die Weihnachtsfeier in seiner lokalen Niederlassung, die nicht seinen Vorstellungen entsprach. Oder etwas vorher kommentierte einer eine Aussage von mir in den Medien, die ihm in den falschen Hals geraten war. Ich nehme solche Mails ernst und schreibe eigentlich immer eine persönliche Antwort darauf, in der ich auf das Problem eingehe, aber auch darauf hinweise, wenn es andere Stellen gibt, an die sich jemand in der Situation wenden sollte. Ich habe nichts gegen solche direkten E-Mails, so lange sie nicht überhand nehmen. Ein Bekannter von mir, ebenfalls CEO in Lateinamerika, sagte mir neulich, er schaue grundsätzlich keine Mails mehr selber an und lasse alles von seiner Assistentin bearbeiten, weil er von solch persönlichem Feedback förmlich überflutet werde.

Wie gehen Sie damit um, dass Sie als CEO einerseits den direkten Kontakt zu Ihren Mitarbeitenden suchen und andererseits in einem global tätigen Unternehmen nie überall vor Ort sein können? Welche Rolle spielen die neuen Medien in diesen globalen Kommunikationsprozessen?

Ich reise sehr viel. Der persönliche Kontakt ist zentral, viel wichtiger als jede elektronische Kommunikation. Wenn ich in einer Länderniederlassung bin, will ich die dortige Führungsmannschaft, die Mitarbeiter, die Kunden und andere wichtige Stakeholders persönlich kennen lernen. Die elektronischen Medien haben aber dennoch eine zentrale Funktion, wir können damit ca. $70 \%$ aller Mitarbeitenden weltweit ansprechen. Für diejenigen ohne eigenen Internetanschluss werden meine CEO-Letters ausgedruckt und in den Fabriken aufgehängt.

Informationen, die für meine Mitarbeiter sehr wichtig sind, publizieren wir nicht nur im Intranet, sondern immer auch per E-Mail. Bei einem Größtunternehmen wie unserem, mit über 110.000 Mitarbeitenden, gibt es sonst kaum eine Möglichkeit, an alle Leute zuverlässig heranzukommen.

\section{Wenn Sie in die Zukunft schauen, welche Chancen und Herausforderungen sehen Sie für Ihre Firma durch das Internet?}

Die digitale Kommunikation wird in Zukunft ein zentrales Werkzeug in der Leitung eines Unternehmens sein. Leider wird sie aber auch im negativen Sinn hohe Bedeutung erhalten: wenn es um Missbrauch, Fälschung, Korruption und böswillige Attacken geht. Wir sehen die große Abhängigkeit von den Informationsnetzwerken beispielsweise bei einem Ausfall eines wichtigen Servers. Eine Firma kann dadurch komplett stillgelegt werden. So ein Zwischenfall kann technisch bedingt sein, er kann durch einen verärgerten Mitarbeiter ausgelöst werden, der sich rächen will, oder auch durch eine kriminelle Organisation. Ein Unternehmen ist diesbezüglich weltweit exponiert und muss unbedingt Vorkehrungen treffen. Man stelle sich vor, wenn in einem peripheren Land übers Internet einen Virus eingeschleust wird, der dazu führt, dass woanders in einer technischen Großanlage, z. B. einem Kraftwerk, ein falsches Ventil geöffnet wird und ein Produktionsprozess außer Kontrolle gelangt. Die weltweit umspannenden Informationstechnologien können ein Segen sein, wenn wir nicht aufpassen aber auch zum Fluch mutieren. 


\subsubsection{Kulturübergreifende Online-Kommunikation}

Technisch gesehen macht es keinen Unterschied, ob ich eine E-Mail an meine Nachbarin sende oder an eine Person auf der anderen Seite des Erdballs. In global tätigen Unternehmen hat sich dadurch der Austausch mit anderen Ländern und Kulturen intensiviert und beschleunigt. Dadurch kommen aber auch Verständigungsschwierigkeiten häufiger vor. Die grundsätzliche Schwierigkeit der Online-Kommunikation, dass man über sein Gegenüber oft wenig weiß, kumuliert sich mit der eigenen Unkenntnis über die fremde Kultur, mit deren Vertreter man kommunizieren sollte. Geschieht dies in einer fremden Sprache, die man nicht gut beherrscht, entstehen zusätzliche Unsicherheiten.

Wie kann man mit dieser Situation umgehen? Auf der Ebene von Marketing und Unternehmenskommunikation wird das Problem oft gelöst, indem man länderspezifische oder regionale Websites und Newsletters konzipiert, die lokale Gegebenheiten aufgreifen können. Aber auch in der persönlichen Zusammenarbeit und im Kundenkontakt über Landesgrenzen hinweg sind interkulturelle Kompetenzen gefragt.

\section{Beispiel}

\section{Zusammenarbeit in globalen Teams}

Global Release IT-Manager, Versicherungsbranche, mittleres Kader, m, 40 Jahre »Ich habe zwei Jahre in England gearbeitet und dort habe ich gesehen, wie Mails gelesen werden, die aus Zürich kommen. Der Ton wird missverstanden. Die Schweizer sind in ihrer Kommunikation relativ direkt, auch untereinander, man sagt, wie es ist. Bei uns gibt es halt keine upper und lower class, in England aber sehr wohl. Und wenn es dann so trocken daherkommt, sind die Engländer zuerst einmal schockiert. Es wird dann leicht vergessen, dass wir keine Muttersprachler sind. Reaktionen wie: »Horror, was wollen die, geht's noch, was ist das für ein Ton...« waren häufig. E-Mail ist sehr schwierig, gerade über Kulturgrenzen hinweg, da werden Sachen missverstanden, die man als glasklar empfindet.

Es gibt einfach die Distanz, eine physische und zum Teil auch eine mentale Distanz, die schwierig zu überbrücken ist. Wir streben in Projekten darum immer an, zuerst einen persönlichen Kontakt herzustellen. Ich gehe nächste Woche aus nur diesem Grund nach New York: um Leute persönlich abzuholen für ein Projekt. Ein persönlicher Kontakt, das macht einen Riesenunterschied. Das habe ich auch mit Leuten aus London gemacht, die ich nach Zürich geholt habe.

Dieser große Unterschied besteht aus mehreren Faktoren. Erstens spürt man sonst die Person einfach nicht: per Telefon nicht, Video geht noch so einigermaßen, aber dort ist es nicht so locker, weil man immer denkt: ich bin jetzt im Fernsehen. Da verhält man sich nicht »normal«.

Man nimmt ja viel mehr wahr, wenn eine Person vor Ort ist, das Nonverbale, auch unbewusst, Mimik, Gestik und all das. Oder auch Nebensäch-
Globale Zusammenarbeit erfordert neue Kompetenzen

Nach dem persönlichen Kontakt werden Medien erst effizient 
Face-to-face-Kontakt erhöht die Verbindlichkeit liches: die Begrüßung, bevor man in den Meetingraum reingeht, evtl. ein gemeinsames Mittagessen. Da spürt man eine Person sehr schnell, und über Medien geht das nicht.

Nach dem persönlichen Kontakt werden die Medien erst wirklich effizient. Man hat eine gewisse Vertrauensbasis und dann kann ich auch mal mit jemandem von Amerika übers Instant Messaging eine Kommunikation haben ohne Problem. Dann sind diese Mittel wieder besser. Anstatt ins Flugzeug zu sitzen, macht man schnell einen Anruf, und auch E-Mail funktioniert besser, wenn man die Person kennt. Wegen des Ziels der $\mathrm{CO}_{2}$-Compliance fliegen wir in unserem Unternehmen momentan aber nur noch, wenn es absolut nötig ist. Da entsteht natürlich sofort eine Grauzone: was ist absolut nötig und was nicht. Muss ich für ein Projekt - den Kickoff - zuerst einmal alle Leute physisch an einem Tisch zusammenhaben oder nicht? Da muss man dann halt abwägen. Aber im Zweifelsfall bin ich definitiv dafür, zuerst einmal den persönlichen Kontakt zu suchen."

\subsection{4 Übersicht:} Kulturübergreifend online kommunizieren

\section{Checkliste: Kulturübergreifend online kommunizieren}

\section{Vor dem Erstkontakt}

- Lassen Sie sich von Bekannten, die Erfahrung mit der entsprechenden Kultur haben, eine Einführung in (geschäfts-) relevante Sitten und Gebräuche geben.

- Versuchen Sie, Grundregeln der Kommunikation im anderen Land (schriftliche und/oder Face-to-face-Kommunikationsrituale) in Erfahrung zu bringen, z. B. notwendige Höflichkeitsformen, übliche Anrede und Grußformel in Texten, Auswahl der Ansprechpartner in Relation zu Ihrer eigenen Person, Wahl des präferierten Kommunikationsmediums für die anstehende Aufgabe.

- Vielleicht gibt es gewisse Stolpersteine oder Missverständnisse, die regelmäßig vorkommen und die Sie ebenfalls im Voraus in Erfahrung bringen können. Entwickeln Sie Strategien, wie Sie diese Stolpersteine umgehen können.

\section{Erstkontakt}

- Falls möglich, wählen Sie für den Erstkontakt eine andere Form als die textbasierte Online-Kommunikation. Ein persönlicher Besuch vor Ort erhöht die Verbindlichkeit der Zusammenarbeit. Ist das nicht möglich, ist ein Telefongespräch oder eine Videokonferenz immer

v noch besser als ein schriftlicher Erstkontakt. Welche Form Sie wäh- 
len, ist natürlich abhängig von der Wichtigkeit der Person oder des Themas, das Sie gemeinsam bearbeiten, und von Ihrer Sprachkompetenz. Es ist in gewissen Fällen sicher einfacher, wenn man eine Anfrage schriftlich formulieren und sich dafür Zeit nehmen kann, als wenn man der Spontanität eines direkten Kontakts ausgeliefert ist.

\section{Im regelmäßigen Austausch}

- Seien Sie hilfsbereit und zuvorkommend und geben Sie v. a. auch positives Feedback, damit Ihr Gegenüber Hinweise darüber hat, welche Aspekte der Zusammenarbeit für Sie gelungen sind.

- Behalten Sie Ihr Wissen über die Kommunikation in der jeweiligen Kultur stets im Hinterkopf. Versuchen Sie, Ihre E-Mails nach diesen Regeln zu formulieren und sich auch an die dort geltenden Höflichkeitsformen zu halten.

- Vermeiden Sie gerade im interkulturellen Austausch unbedingt Doppeldeutigkeiten und Ironie. Ausnahme: Sie kennen Ihr Gegenüber sehr gut oder sehr lange und haben zusammen eine Kommunikationskultur aufgebaut, die das zulässt.

- Bedenken Sie, dass regelmäßige Videokonferenzen für den Austausch nicht immer den gewünschten Effekt haben - sie können statt zu besserer Verständigung zu stärkerer Polarisierung zwischen den beiden Parteien führen ( Kap. 3.3.3).

- Fassen Sie E-Mails nicht zu knapp, dadurch entsteht zusätzlicher Interpretationsspielraum auf der anderen Seite. Erklären Sie Ihre Anliegen explizit.

- Vereinbaren Sie klare Ziele und setzen Sie verbindliche Termine. So haben Sie die Möglichkeit, beim Gegenüber zu einem bestimmten Zeitpunkt nachzuhaken, ohne als unhöflich zu gelten.

- Wenn Sie länger als erwartet keine Antwort erhalten, greifen Sie zum Telefon und fragen Sie mündlich nach.

- Ergänzen Sie Ihre Online-Kontakte grundsätzlich immer wieder durch Kommunikation via andere Kanäle.

\subsection{Zusammenfassung: Die Eigenschaften der Online-Kommunikation und ihre Vor- und Nachteile}

Im textbasierten Online-Austausch (z. B. E-Mail, Foren, Blogs, Chat) besteht ein erhöhtes Potenzial für Missverständnisse, weil die Beziehungsaspekte der Botschaft (para- und nonverbale Kommunikation) nur eingeschränkt übermittelt werden können.

Die fehlenden Informationen über den Empfänger einer Nachricht führen beim Sender zu Enthemmung und größerer Offenheit. Die Anonymität erhöht nicht nur die Bereitschaft, Persönliches preiszugeben, sondern begünstigt auch unangemessenes oder aggressives Verhalten. 
Textbasierte Kommunikation ist effizienter in der Vermittlung von detaillierten Inhalten, Bilder können Emotionen und Zusammenhänge deutlich machen.

Das digitale Datenformat beeinflusst kommunikative Prozesse stark und hat ambivalente Folgen:

- Informationen werden schnell und kostengünstig vervielfältigt (und lösen dadurch auch Überlastungsgefühle aus).

- Informationen können einfach aufgefunden und kontrolliert werden (auch von Unbefugten).

- Der bessere Informationsfluss in hierarchischen Gefügen führt zu mehr Transparenz (aber auch zu inadäquatem Verhalten).

- Der Teilnehmerkreis kann problemlos erweitert werden (und dadurch auch die Arbeitslast).

\section{Weiterführende Literatur}

Döring, N. (2003). Sozialpsychologie des Internet. Die Bedeutung des Internets für Kommunikationsprozesse, Identitäten, soziale Beziehungen und Gruppen. Göttingen: Hogrefe. Der Klassiker zum Thema Psychologie und Internet aus wissenschaftlicher Sicht. 